\title{
Asymptotic model of linearly visco-elastic Kelvin-Voigt type plates via Trotter theory
}

Yotsawat Terapabkajornded ${ }^{1}$, Somsak Orankitjaroen ${ }^{1,3^{*}}$ (I) and Christian Licht ${ }^{1,2,3}$

\section{"Correspondence:}

somsak.ora@mahidol.ac.th

${ }^{1}$ Department of Mathematics,

Faculty of Science, Mahidol

University, Bangkok, Thailand

${ }^{3}$ Centre of Excellence in

Mathematics, CHE, Bangkok,

Thailand

Full list of author information is

available at the end of the article

\section{Springer}

\begin{abstract}
We confirm the study (Licht in C. R., Méc. 341:697-700, 2013) devoted to the quasi-static response for a visco-elastic Kelvin-Voigt plate whose thickness goes to zero. For each thickness parameter, the quasi-static response is given by a system of partial differential equations with initial and boundary conditions. Reformulating scaled systems into a family of evolution equations in Hilbert spaces of possible states with finite energy, we use Trotter theory of convergence of semi-groups of linear operators to identify the asymptotic behavior of the system. The asymptotic model we obtain and the genuine one have the same structure except an occurrence of a new state variable. Eliminating the new state variable from our asymptotic model leads to the asymptotic model in (Licht in C. R., Méc. 341:697-700, 2013) which involves an integro-differential system.
\end{abstract}

MSC: $74 \mathrm{~B} 99$

Keywords: Asymptotic model; Thin visco-elastic plates; Kelvin-Voigt visco-elasticity; Trotter theory

\section{Introduction}

In a recent study [2], Licht and Weller promoted an old but not so well-known convergence tool, namely Trotter theory of convergence of semi-groups of linear operators acting on variable Hilbert spaces, in determining the asymptotic modeling in physics of continuous media. They provided various asymptotic models through the lens of Trotter theory as a comparison to other classical methods. One of the models mentioned is a reduction of the dimension problem on thin linear visco-elastic Kelvin-Voigt type plates. Licht [1] studied this problem before in 2013 and derived the asymptotic model with Laplace transform technique. He found that the mechanical behavior of the limit model is no longer of Kelvin-Voigt type, because a term of fading memory appears like in the homogenization problem. However, with Trotter theory of convergence, Licht and Weller suggested that the mechanical behaviors of limit and genuine models are the same except for the appearance of a new state variable. It is well known that to have the same structure in both limit and genuine models is useful for numerical computations.

In this study we aim to justify and confirm their suggestion. We reconsider a reduction of the dimension problem of thin linearly visco-elastic Kelvin-Voigt plates in Sect. 2. By defining a small parameter $\varepsilon$, referred to as the thickness of the plate, each problem is expressed as an initial-boundary value problem (2.1). Under suitable assumptions, we

(c) The Author(s) 2019. This article is distributed under the terms of the Creative Commons Attribution 4.0 International License (http://creativecommons.org/licenses/by/4.0/), which permits unrestricted use, distribution, and reproduction in any medium, provided you give appropriate credit to the original author(s) and the source, provide a link to the Creative Commons license, and indicate if changes were made. 
rescale the problems and reformulate them in terms of a family of transient problems. In Sect. 3 we discuss our convergence tool, that is, Trotter theory of the convergence of semigroups of linear operators. We then follow a Trotter theory approach by letting $\varepsilon$ tend to zero to derive the limit model in Sect. 4. As will be seen in the last section, the limit model contains an additional state variable but with the structure like that of the original one. Eliminating the new state variable from the limit model recovers an additional term of fading memory in the limit model derived by the Laplace transform technique, which implies integro-differential equations involving partial derivatives of the field of displacement.

\section{Setting the problem}

Customarily, we assimilate the physical Euclidean space to $\mathbb{R}^{3}$; the orthonormal basis of which is denoted by $\left\{e_{1}, e_{2}, e_{3}\right\}$, and for all $\xi=\left(\xi_{1}, \xi_{2}, \xi_{3}\right)$ in $\mathbb{R}^{3}, \widehat{\xi}:=\left(\xi_{1}, \xi_{2}\right)$. We will study the quasi-static response of a thin linearly visco-elastic Kelvin-Voigt plate subjected to a given load. Like the problem setting in [1], a thin linearly visco-elastic Kelvin-Voigt plate occupies a domain $\Omega^{\varepsilon}:=\omega \times(-\varepsilon, \varepsilon)$, where $\omega$ is a bounded domain in $\mathbb{R}^{2}$ with a Lipschitzcontinuous boundary $\partial \omega$ and $\varepsilon$ is the small thickness of the plate. The upper, lower surfaces $\omega \times\{ \pm \varepsilon\}$ and the lateral part of the plate $\omega \times(-\varepsilon, \varepsilon)$ are referred to as $\Gamma_{ \pm}^{\varepsilon}$ and $\Gamma_{\text {lat }}^{\varepsilon}$, respectively. The plate is clamped along a portion of the lateral part $\Gamma_{\mathrm{D}}^{\varepsilon}:=\gamma_{\mathrm{D}} \times(-\varepsilon, \varepsilon)$ where $\gamma_{D}$ is of positive length. Moreover, it is subjected to body forces of density $f^{\varepsilon}$ and surface forces of density $g^{\varepsilon}$ on the upper, lower surfaces together with the rest of its lateral part $\Gamma_{\mathrm{N}}^{\varepsilon}:=\partial \Omega^{\varepsilon} \backslash \Gamma_{\mathrm{D}}^{\varepsilon}$ during the time interval $[0, T]$. We denote $\Gamma_{\mathrm{N} \pm}^{\varepsilon}=\Gamma_{\mathrm{N}}^{\varepsilon} \cap \Gamma_{ \pm}^{\varepsilon}$ and $\Gamma_{\mathrm{N}, \text { lat }}^{\varepsilon}=\Gamma_{\mathrm{N}}^{\varepsilon} \cap \Gamma_{\text {lat }}^{\varepsilon}$.

The problem of determining the quasi-static evolution of the plate involves the parameter $\varepsilon$ of data and the equations satisfied by the fields of displacement $u^{\varepsilon}$ and stress $\sigma^{\varepsilon}$ are:

$$
\begin{cases}-\operatorname{div} \sigma^{\varepsilon}=f^{\varepsilon} & \text { in } \Omega^{\varepsilon} \times(0, T), \\ \sigma^{\varepsilon}=a^{\varepsilon} e\left(u^{\varepsilon}\right)+b^{\varepsilon} e\left(\dot{u}^{\varepsilon}\right) & \text { in } \Omega^{\varepsilon} \times(0, T), \\ e_{i j}\left(u^{\varepsilon}\right):=\left(\partial_{i} u_{j}^{\varepsilon}+\partial_{j} u_{i}^{\varepsilon}\right) / 2, & \\ \sigma^{\varepsilon} n^{\varepsilon}=g^{\varepsilon} & \text { on } \Gamma_{\mathrm{N}}^{\varepsilon} \times(0, T), u^{\varepsilon}=0 \text { on } \Gamma_{\mathrm{D}}^{\varepsilon} \times(0, T), \\ u^{\varepsilon}(\cdot, 0)=u_{0}^{\varepsilon} & \text { in } \Omega^{\varepsilon},\end{cases}
$$

where $e\left(u^{\varepsilon}\right), n^{\varepsilon}, a^{\varepsilon}$ and $b^{\varepsilon}$ are the linearized strain tensor, the outward unit normal vector, the elasticity and viscosity tensor fields, respectively, while the upper dot represents the time derivative.

To obtain a simpler but precise enough model we study the quasi-static response for the plate as its thickness tends to zero. Following [1,3], first we rescale the domain $\Omega^{\varepsilon}$ into a fixed domain $\Omega:=\omega \times(-1,1)$ through the mapping $\pi^{\varepsilon}$ :

$$
x=\left(\widehat{x}, x_{3}\right) \in \bar{\Omega} \quad \mapsto \quad \pi^{\varepsilon} x=\left(\widehat{x}, \varepsilon x_{3}\right)=: x^{\varepsilon} \in \bar{\Omega}^{\varepsilon} .
$$

Next we add two hypotheses to the data. Hypothesis $\left(\mathrm{H}_{1}\right)$ is on the real loading which has to be connected to fixed quantities defined on $\bar{\Omega}$, they concern their intensity and 
horizontality:

$$
\left(\mathrm{H}_{1}\right):\left\{\begin{array}{l}
\exists(f, g) \in C^{0,1}\left([0, T] ; L^{2}\left(\Omega ; \mathbb{R}^{3}\right)\right) \times C^{1,1}\left([0, T] ; L^{2}\left(\Gamma_{\mathrm{N}} ; \mathbb{R}^{3}\right)\right) \quad \text { such that } \\
\widehat{f^{\varepsilon}}\left(\pi^{\varepsilon} x, t\right)=\varepsilon \widehat{f}(x, t), \quad f_{3}^{\varepsilon}\left(\pi^{\varepsilon} x, t\right)=\varepsilon^{2} f_{3}(x, t), \quad \forall(x, t) \in \Omega \times[0, T], \\
\widehat{g^{\varepsilon}}\left(\pi^{\varepsilon} x, t\right)=\varepsilon^{2} \widehat{g}(x, t), \quad g_{3}^{\varepsilon}\left(\pi^{\varepsilon} x, t\right)=\varepsilon^{3} g_{3}(x, t), \quad \forall(x, t) \in \Gamma_{\mathrm{N} \pm} \times[0, T], \\
g_{3}^{\varepsilon}\left(\pi^{\varepsilon} x, t\right)=\varepsilon^{2} g_{3}(x, t), \quad \forall(x, t) \in \Gamma_{\mathrm{N}, \text { lat }} \times[0, T],
\end{array}\right.
$$

while hypothesis $\left(\mathrm{H}_{2}\right)$ is on the elasticity and viscosity tensors:

$$
\left(\mathrm{H}_{2}\right):\left\{\begin{array}{l}
a^{\varepsilon}\left(\pi^{\varepsilon} x\right)=a(x), \quad b^{\varepsilon}\left(\pi^{\varepsilon} x\right)=b(x) \quad \text { with } a, b \in L^{\infty}\left(\Omega ; \operatorname{Lin}\left(\mathbb{S}^{3}\right)\right), \quad \text { and } \\
\exists \kappa_{A}, \kappa_{a}>0: \kappa_{a}|e|^{2}<a(x) e \cdot e<\kappa_{A}|e|^{2}, \quad \forall e \in \mathbb{S}^{3}, \quad \text { a.e. } x \in \Omega, \\
\exists \kappa_{B}, \kappa_{b}>0: \kappa_{b}|e|^{2}<b(x) e \cdot e<\kappa_{B}|e|^{2}, \quad \forall e \in \mathbb{S}^{3}, \quad \text { a.e. } x \in \Omega,
\end{array}\right.
$$

where $\operatorname{Lin}\left(\mathbb{S}^{3}\right)$ denotes the space of linear mapping from $\mathbb{S}^{3}$ into $\mathbb{S}^{3}, \mathbb{S}^{3}$ being the space of $3 \times 3$ symmetric matrices. It will be convenient to write $\mathbb{S}^{3}=\widehat{\mathbb{S}} \oplus \mathbb{S}^{\perp}$ with $\widehat{\mathbb{S}}:=\left\{e \in \mathbb{S}^{3} ; e_{i 3}=\right.$ $0,0 \leq i \leq 3\}, \mathbb{S}^{\perp}:=\left\{e \in \mathbb{S}^{3} ; e_{\alpha \beta}=0,1 \leq \alpha, \beta \leq 2\right\}$, and to denote the projection of $e$ on $\widehat{\mathbb{S}}$ and $\mathbb{S}^{\perp}$ by $\widehat{e}$ and $e^{\perp}$, respectively. Then one associates a scaled displacement $u_{\varepsilon}=S(\varepsilon) u^{\varepsilon}$, defined on $\bar{\Omega} \times[0, T]$, with the true physical displacement $u^{\varepsilon}$, defined on $\bar{\Omega}^{\varepsilon} \times[0, T]$, by

$$
\widehat{u^{\varepsilon}}\left(x^{\epsilon}, t\right)=\varepsilon \widehat{u_{\varepsilon}}(x, t), \quad u_{3}^{\varepsilon}\left(x^{\varepsilon}, t\right)=\left(u_{\varepsilon}\right)_{3}(x, t) \quad \forall\left(x^{\varepsilon}, t\right) \in \bar{\Omega}^{\varepsilon} \times[0, T] .
$$

Using hypotheses $\left(\mathrm{H}_{1}\right)$ and $\left(\mathrm{H}_{2}\right)$ and the scaling of displacement, we can formulate the initial-boundary value problem (2.1) in terms of the scaled variational problem

$$
\left(\mathcal{P}_{\varepsilon}\right):\left\{\begin{array}{l}
u_{\varepsilon} \in C^{1,1}\left([0, T] ; H_{\varepsilon}\right), \quad u_{\varepsilon}(\cdot, 0)=u_{\varepsilon}^{0}:=S(\varepsilon) u_{0}^{\varepsilon} ; \\
\int_{\Omega}\left[a(x) e\left(\varepsilon, u_{\varepsilon}\right)(x, t)+b e\left(\varepsilon, \dot{u}_{\varepsilon}\right)(x, t)\right] \cdot e(\varepsilon, v)(x) d x \\
=\int_{\Omega} f(x, t) \cdot v(x) d x+\int_{\Gamma_{\mathrm{N}}} g(x, t) \cdot v(x) d \mathcal{H}_{2} \quad \forall v \in H_{\varepsilon}
\end{array}\right.
$$

where $H_{\varepsilon}$ is the subspace of $H^{1}\left(\Omega, \mathbb{R}^{3}\right)$ whose elements have a vanishing trace on $\Gamma_{\mathrm{D}}$, $e_{\alpha \beta}(\varepsilon, v)=e_{\alpha, \beta}(v), e_{\alpha 3}(\varepsilon, v)=\varepsilon^{-1} e_{\alpha 3}(v), 1 \leq \alpha \beta \leq 2, e_{33}(\varepsilon, v)=\varepsilon^{-2} e_{33}(v)$ and $\mathcal{H}_{2}$ is the twodimensional Hausdorff measure. Subspace $H_{\varepsilon}$ is equipped with the inner product:

$$
(u, v)_{H_{\varepsilon}}=\int_{\Omega} a e(\varepsilon, u) \cdot e(\varepsilon, v) d x \quad \forall u, v \in H_{\varepsilon}
$$

To deal with non-vanishing external loadings, it suffices to split $u_{\varepsilon}$ into $u_{\varepsilon}^{e}+u_{\varepsilon}^{r}$, where $u_{\varepsilon}^{e}$ solves a static problem associated with the evolution problem under consideration and involving the surface loading only. Then $u_{\varepsilon}^{r}$ does solve an evolution equation with a second member which is a continuous function of $u_{\varepsilon}^{e}$ and hence a continuous function of the loading. The static problem is

$$
\left(\mathcal{P}_{\varepsilon}^{e}\right):\left\{\begin{array}{l}
u_{\varepsilon}^{e}(t) \in H_{\varepsilon} \\
\int_{\Omega} a e\left(\varepsilon, u_{\varepsilon}^{e}(t)\right) \cdot e(\varepsilon, v) d x=\int_{\Gamma_{\mathrm{N}}} g(x, t) \cdot v(x) d \mathcal{H}_{2} \quad \forall v \in H_{\varepsilon}
\end{array}\right.
$$


which has a unique solution by the Lax-Milgram lemma. As $g \mapsto u_{\varepsilon}^{e}$ is linear continuous from $L^{2}\left(\Gamma_{\mathrm{N}} ; \mathbb{R}^{3}\right)$ to $H_{\varepsilon}$, we have $u_{\varepsilon}^{e}$ in $C^{1,1}\left([0, T] ; H_{\varepsilon}\right)$ The evolution problem is

$$
\left(\mathcal{P}_{\varepsilon}^{r}\right):\left\{\begin{array}{l}
u_{\varepsilon}^{r} \in C^{1,1}\left([0, T] ; H_{\varepsilon}\right) ; \\
\int_{\Omega}\left[a e\left(\varepsilon, u_{\varepsilon}^{r}\right)+b e\left(\varepsilon, \dot{u}_{\varepsilon}^{r}\right)\right] \cdot e(\varepsilon, v) d x \\
\quad=\int_{\Omega} f \cdot v d x-\int_{\Omega} b e\left(\varepsilon, \dot{u}_{\varepsilon}^{e}\right) \cdot e(\varepsilon, v) d x \quad \forall v \in H_{\varepsilon},
\end{array}\right.
$$

which can be reformulated in terms of

$$
\left\{\begin{array}{l}
\frac{d u_{\varepsilon}^{r}}{d t}-A_{\varepsilon} u_{\varepsilon}^{r}=q_{\varepsilon} \quad \text { in } H_{\varepsilon} \\
u_{\varepsilon}^{r}(0)=u_{\varepsilon}^{r 0}:=u_{\varepsilon}^{0}-u_{\varepsilon}^{e}(0)
\end{array}\right.
$$

with $D\left(A_{\varepsilon}\right)=H_{\varepsilon}$,

$$
\begin{aligned}
& A_{\varepsilon} u_{\varepsilon}=w_{\varepsilon} \in H_{\varepsilon} ; \quad \int_{\Omega}\left[a e\left(\varepsilon, u_{\varepsilon}\right)+b e\left(\varepsilon, w_{\varepsilon}\right)\right] \cdot e(\varepsilon, v) d x=0 \quad \forall v \in H_{\varepsilon} \\
& \left(q_{\varepsilon}(t), v\right)_{H_{\varepsilon}}=\int_{\Omega} f(x, t) \cdot v(x) d x-\int_{\Omega} e\left(\varepsilon, \dot{u}^{e}(t)\right) \cdot e(\varepsilon, v) d x \quad \forall v \in H_{\varepsilon} .
\end{aligned}
$$

Clearly $A_{\varepsilon}$ is bounded, selfadjoint and $m$-dissipative so that the evolution equation (2.2) has a unique solution $u_{\varepsilon}^{r}$ in $C^{1,1}\left([0, T] ; H_{\varepsilon}\right)$.

The crucial point to prove that the asymptotic model has the same structure as the genuine one is to apply the tool of some convergence which is a not so well-known result, involving two fields, in reduction of dimension similar to two-scale convergence in periodic homogenization. The proposed asymptotic models of $\left(\mathcal{P}_{\varepsilon}^{e}\right)$ and $\left(\mathcal{P}_{\varepsilon}^{r}\right)$ will be given by the following problems:

$$
\left(\mathcal{P}^{e}\right):\left\{\begin{array}{l}
U^{e}:=\left(u^{e}, u^{e 1}\right) \in H \\
\int_{\Omega} a\left(\widehat{e}\left(u^{e}\right)+\partial_{3} u^{e 1} \otimes_{s} e_{3}\right) \cdot\left(\widehat{e}(v)+\partial_{3} v^{1} \otimes_{s} e_{3}\right) d x=\int_{\Gamma_{\mathrm{N}}} g \cdot v d \mathcal{H}_{2} \\
\forall V=\left(v, v^{1}\right) \in H,
\end{array}\right.
$$

set in a Hilbert space $H:=V_{\mathrm{KL}}(\Omega) \times H^{1}\left(-1,1 ; L^{2}\left(\omega ; \mathbb{R}^{3}\right)\right) / L^{2}\left(\omega ; \mathbb{R}^{3}\right)$, with $V_{\mathrm{KL}}=\{u \in$ $\left.H_{\Gamma_{\mathrm{D}}}^{1}\left(\Omega ; \mathbb{R}^{3}\right) ; e(u)^{\perp}=0\right\}$, where $H_{\Gamma_{\mathrm{D}}}^{1}\left(\Omega ; \mathbb{R}^{3}\right)$ is the subspace of $H^{1}\left(\Omega ; \mathbb{R}^{3}\right)$ whose elements have a vanishing trace on $\Gamma_{\mathrm{D}}$, and equipped with the inner product:

$$
(U, V)_{H}=\int_{\Omega} a\left(\widehat{e}(u)+\partial_{3} u^{1} \otimes_{s} e_{3}\right) \cdot\left(\widehat{e}(v)+\partial_{3} v^{1} \otimes_{s} e_{3}\right) d x
$$

and

$$
\left(\mathcal{P}^{r}\right):\left\{\begin{array}{l}
U^{r}:=\left(u^{r}, u^{r 1}\right) \in C^{1,1}([0, T] ; H) ; \\
\int_{\Omega}\left[a\left(\widehat{e}\left(u^{r}\right)+\partial_{3} u^{r 1} \otimes_{s} e_{3}\right)+b\left(\widehat{e}\left(\dot{u}^{r}\right)+\partial_{3} \dot{u}^{r 1} \otimes_{s} e_{3}\right)\right] \cdot\left(\widehat{e}(v)+\partial_{3} v^{1} \otimes_{s} e_{3}\right) d x \\
\quad=\int_{\Omega} f \cdot v d x-\int_{\Omega} b\left(\widehat{e}\left(\dot{u}^{e}\right)+\partial_{3} \dot{u}^{e 1} \otimes_{s} e_{3}\right) \cdot\left(\widehat{e}(v)+\partial_{3} v^{1} \otimes_{s} e_{3}\right) d x \\
\forall V=\left(v, v^{1}\right) \in H,
\end{array}\right.
$$


which can be reformulated in terms of

$$
\left\{\begin{array}{l}
\frac{d U^{r}}{d t}-A U^{r}=Q \quad \text { in } H \\
U^{r}(0)=U^{r 0}:=U^{0}-U^{e}(0)
\end{array}\right.
$$

with $D(A)=H$,

$$
\begin{aligned}
A U=W= & \left(w, w^{1}\right) \in H ; \int_{\Omega}\left[a\left(\widehat{e}(u)+\partial_{3} u^{1} \otimes_{s} e_{3}\right)+b\left(\widehat{e}(w)+\partial_{3} w^{1} \otimes_{s} e_{3}\right)\right] \\
\cdot(\widehat{e}(v) & \left.+\partial_{3} v^{1} \otimes_{s} e_{3}\right) d x=0 \quad \forall V=\left(v, v^{1}\right) \in H, \\
(Q(t), V)_{H}= & -\int_{\Omega}\left(\widehat{e}\left(\dot{u}^{e}(t)\right)+\partial_{3} \dot{u}^{e 1}(t) \otimes_{s} e_{3}\right) \cdot\left(\widehat{e}(v)+\partial_{3} v^{1} \otimes_{s} e_{3}\right) d x \\
& +\int_{\Omega} f(x, t) \cdot v(x) d x \quad \forall V=\left(v, v^{1}\right) \in H .
\end{aligned}
$$

Obviously $A$ is bounded, selfadjoint and $m$-dissipative. Problem $\left(\mathcal{P}^{e}\right)$ has a unique solution $U^{e}$ by the Lax-Milgram lemma. and the evolution problem (2.3) has a unique solution $U^{r}$ in $C^{1,1}([0, T] ; H)$.

At this point, we have solutions $\left(u_{\varepsilon}^{e}\right),\left(u_{\varepsilon}^{r}\right), U^{e}$, and $U^{r}$ to the problems $\left(\mathcal{P}_{\varepsilon}^{e}\right),\left(\mathcal{P}_{\varepsilon}^{r}\right),\left(\mathcal{P}^{e}\right)$, and $\left(\mathcal{P}^{r}\right)$, respectively. We claim that the sequences $\left(u_{\varepsilon}^{e}\right),\left(u_{\varepsilon}^{r}\right)$ converge to $U^{e}, U^{r}$ in the sense of Trotter. Before we prove our claim, let us discuss this type of convergence in detail.

\section{Trotter theory of convergence of semi-groups of linear operators}

In the framework of Trotter theory of convergence of semi-groups of linear operators, we have a family of evolution equations

$$
\left(\mathcal{P}_{n}\right):\left\{\begin{array}{l}
\frac{d u_{n}}{d t}-A_{n} u_{n}=q_{n} \quad \text { in } H_{n} \\
u_{n}(0)=u_{n}^{0}
\end{array}\right.
$$

governed by a sequence of $m$-dissipative operators $A_{n}$, with domains $D\left(A_{n}\right)$, defined in a sequence of Hilbert spaces $\left(H_{n}\right)$ with norms $\|\cdot\|_{H_{n}}$, while initial data $u_{n}^{0}$ and $q_{n}$ are in $D\left(A_{n}\right) \times C^{0,1}\left([0, T] ; H_{n}\right)$. Each problem $\mathcal{P}_{n}$ thus has a unique solution in $C^{1,1}\left([0, T] ; H_{n}\right) \cap$ $C^{0,1}\left([0, T] ; D\left(A_{n}\right)\right)$. On the other hand we have an evolution equation

$$
(\mathcal{P}):\left\{\begin{array}{l}
\frac{d u}{d t}-A u=q \quad \text { in } H \\
u(0)=u^{0},
\end{array}\right.
$$

governed by an $m$-dissipative operator $A$, with domain $D(A)$, defined in a Hilbert spaces $H$ with a norm $\|\cdot\|_{H}$, while initial data $u^{0}$ and $q$ in $D(A) \times C^{0,1}([0, T] ; H)$. Problem $(\mathcal{P})$ also has a unique solution in $C^{1,1}([0, T] ; H) \cap C^{0,1}([0, T] ; D(A))$. The nature of the spaces $H_{n}$ and $H$ may be very different, but the sequence of spaces $H_{n}$ has to converge to $H$. This requirement paves the way to introduce a kind of sequence of representative operators $\mathrm{P}_{n}$ between $H$ and $H_{n}$. 
Definition 1 We say that a sequence of Hilbert spaces $H_{n}$ approximates a Hilbert space $H$ in the sense of Trotter, if there exists a representative operator $\mathrm{P}_{n} \in \mathcal{L}\left(H ; H_{n}\right)$ satisfying two conditions of uniform continuity $\left(T_{1}\right)$ and good energetic representation $\left(T_{2}\right)$ :

$\left(T_{1}\right) \exists C>0$ such that $\left\|\mathrm{P}_{n} u\right\|_{H_{n}} \leq C\|u\|_{H}, \forall u \in H, \forall n$,

$\left(T_{2}\right) \lim _{n \rightarrow \infty}\left\|\mathrm{P}_{n} u\right\|_{H_{n}}=\|u\|_{H}, \forall u \in H$,

where $\mathcal{L}\left(H ; H_{n}\right)$ is a space of all continuous linear mappings from $H$ into $H_{n}$.

Definition 2 A sequence $\left(u_{n}\right)$ in $H_{n}$ converges toward an element $u$ in $H$ in the sense of Trotter if and only if $\lim _{n \rightarrow \infty}\left\|\mathrm{P}_{n} u-u_{n}\right\|_{H_{n}}=0$.

The fundamental result of Trotter theory of convergence of semi-groups of linear operators is the following.

Theorem 1 Let $u_{n}, u$ be the solutions of $\left(\mathcal{P}_{n}\right)$ and $(\mathcal{P})$, respectively, if

(i) $\left\|\mathrm{P}_{n} u^{0}-u_{n}^{0}\right\|_{H_{n}} \rightarrow 0$,

(ii) $\int_{0}^{T}\left\|\mathrm{P}_{n} q(t)-q_{n}(t)\right\|_{H_{n}} d t \rightarrow 0$,

(iii) $\forall y \in X$, dense in $H,\left\|\mathrm{P}_{n}(I-A)^{-1} y-\left(I-A_{n}\right)^{-1} \mathrm{P}_{n} y\right\|_{H_{n}} \rightarrow 0$

then, uniformly on $[0, T],\left\|\mathrm{P}_{n} u(t)-u_{n}(t)\right\|_{H_{n}} \rightarrow 0$ and $\left\|u_{n}(t)\right\|_{H_{n}} \rightarrow\|u(t)\|_{H}$.

Now we are ready to prove our assertion.

\section{A convergence result}

First we have to show that the sequence of Hilbert spaces $H_{\varepsilon}$ approximate the Hilbert space $H$ in the sense of Trotter. Let a representative operator $\mathrm{P}_{\varepsilon}$ be defined by $u_{\varepsilon}^{\prime}=\mathrm{P}_{\varepsilon} U$ for all $U=\left(u, u^{1}\right)$ that satisfies

$$
\int_{\Omega} a e\left(\varepsilon, u_{\varepsilon}^{\prime}\right) \cdot e(\varepsilon, v) d x=\int_{\Omega} a\left(\widehat{e}(u)+\partial_{3} u^{1} \otimes_{s} e_{3}\right) \cdot e(\varepsilon, v) d x \quad \forall v \in H_{\varepsilon} .
$$

The operator $\mathrm{P}_{\varepsilon}$ satisfies $\left(T_{1}\right)$ because

$$
\left\|\mathrm{P}_{\varepsilon} U\right\|_{H_{\varepsilon}}^{2}=\int_{\Omega} a\left(\widehat{e}(u)+\partial_{3} u^{1} \otimes_{s} e_{3}\right) \cdot e\left(\varepsilon, u_{\varepsilon}^{\prime}\right) d x \leq C\|U\|_{H}\left\|\mathrm{P}_{\varepsilon} U\right\|_{H_{\varepsilon}} .
$$

Now choose $v=u_{\varepsilon}^{\prime}$ in (4.1). The sequences $\left(u_{\varepsilon}^{\prime}\right)$ and $e\left(\varepsilon, u_{\varepsilon}^{\prime}\right)$ are bounded in $H_{\Gamma_{\mathrm{D}}}^{1}\left(\Omega ; \mathbb{R}^{3}\right)$ and $L^{2}\left(\Omega ; \mathbb{S}^{3}\right)$, respectively. Then there exist $\left(u^{\prime}, \kappa^{\prime}\right)$ in $H_{\Gamma_{\mathrm{D}}}^{1}\left(\Omega ; \mathbb{R}^{3}\right) \times L^{2}\left(\Omega ; \mathbb{S}^{3}\right)$ such that $\left(u_{\varepsilon}^{\prime}, e\left(\varepsilon, u_{\varepsilon}^{\prime}\right)\right)$ weakly converge toward $\left(u^{\prime}, \kappa^{\prime}\right)$ in $H_{\Gamma_{\mathrm{D}}}^{1}\left(\Omega ; \mathbb{R}^{3}\right) \times L^{2}\left(\Omega ; \mathbb{S}^{3}\right)$. It follows that $\widehat{e}\left(\varepsilon, u_{\varepsilon}^{\prime}\right)$ weakly converge to $\widehat{e}\left(u^{\prime}\right)=\widehat{\kappa^{\prime}}$ in $L^{2}\left(\Omega ; \mathbb{S}^{3}\right)$. Now we are in the position to introduce in reduction of dimension problem a kind of convergence involving a couple of limit fields similar to the two-scaled convergence in periodic homogenization problem. There exists $u^{\prime 1}$ in $H^{1}\left(-1,1 ; L^{2}\left(\omega ; \mathbb{R}^{3}\right)\right) / L^{2}\left(\omega ; \mathbb{R}^{3}\right)$ such that $e\left(\varepsilon, u_{\varepsilon}^{\prime}\right)^{\perp}$ weakly converge to $\kappa^{\prime \perp}=\partial_{3} u^{\prime 1} \otimes_{s} e_{3}$ in $L^{2}\left(\Omega ; \mathbb{S}^{3}\right)$. Hence, $\kappa^{\prime}=\widehat{e}\left(u^{\prime}\right)+\partial_{3} u^{\prime 1} \otimes_{s} e_{3}$. Next we choose $v=\left(w+\varepsilon\left(\widehat{w}^{1}, \varepsilon w_{3}^{1}\right)\right)$ where $w$ in $V_{\mathrm{KL}}, w^{1}$ in $H^{1}\left(-1,1 ; L^{2}\left(\omega ; \mathbb{R}^{3}\right)\right) / L^{2}\left(\omega ; \mathbb{R}^{3}\right)$ in (4.1). As $\varepsilon$ tends to zero, (4.1) becomes

$$
\left(U^{\prime}, W\right)_{H}=(U, W)_{H} \quad \forall W \in H,
$$

where $U^{\prime}=\left(u^{\prime}, u^{\prime 1}\right), U=\left(u, u^{1}\right)$, and $W=\left(w, w^{1}\right)$. It follows that $U^{\prime}=U$. 
Hence,

$$
\begin{aligned}
\lim _{\varepsilon \rightarrow 0}\left\|\mathrm{P}_{\varepsilon} U\right\|_{H_{\varepsilon}}^{2} & =\lim _{\varepsilon \rightarrow 0} \int_{\Omega} a e\left(\varepsilon, u_{\varepsilon}^{\prime}\right) \cdot e\left(\varepsilon, u_{\varepsilon}^{\prime}\right) d x \\
& =\lim _{\varepsilon \rightarrow 0} \int_{\Omega} a\left(\widehat{e}(u)+\partial_{3} u^{1} \otimes_{s} e_{3}\right) \cdot e\left(\varepsilon, u_{\varepsilon}^{\prime}\right) d x \\
& =\int_{\Omega} a\left(\widehat{e}(u)+\partial_{3} u^{1} \otimes_{s} e_{3}\right) \cdot\left(\widehat{e}\left(u^{\prime}\right)+\partial_{3} u^{\prime 1} \otimes_{s} e_{3}\right) d x \\
& =\int_{\Omega} a\left(\widehat{e}(u)+\partial_{3} u^{1} \otimes_{s} e_{3}\right) \cdot\left(\widehat{e}(u)+\partial_{3} u^{1} \otimes_{s} e_{3}\right) d x=\|U\|_{H}^{2} .
\end{aligned}
$$

That is, operator $\mathrm{P}_{\varepsilon}$ satisfies $\left(T_{2}\right)$. Thus, the sequence of Hilbert spaces $H_{\varepsilon}$ approximates the Hilbert space $H$ in the sense of Trotter.

To prove our convergence result, the following lemma is important.

Lemma 1 For all $z_{\varepsilon}$ in $H_{\varepsilon}, Z$ in $H$,

$$
\left\|\mathrm{P}_{\varepsilon} Z-z_{\varepsilon}\right\|_{H_{\varepsilon}} \rightarrow 0 \quad \Longleftrightarrow \quad e\left(\varepsilon, z_{\varepsilon}\right) \rightarrow \widehat{e}(z)+\partial_{3} z^{1} \otimes_{s} e_{3} \quad \text { in } L^{2}\left(\Omega ; \mathbb{S}^{3}\right)
$$

Proof Observe that

$$
\begin{aligned}
\left\|\mathrm{P}_{\varepsilon} Z-z_{\varepsilon}\right\|_{H_{\varepsilon}}^{2} & =\left\|\mathrm{P}_{\varepsilon} Z\right\|_{H_{\varepsilon}}^{2}-2\left(\mathrm{P}_{\varepsilon} Z, z_{\varepsilon}\right)_{H_{\varepsilon}}+\left\|z_{\varepsilon}\right\|_{H_{\varepsilon}}^{2} \\
& =\left\|\mathrm{P}_{\varepsilon} Z\right\|_{H_{\varepsilon}}^{2}-2 \int_{\Omega} a e\left(\varepsilon, z_{\varepsilon}^{\prime}\right) \cdot e\left(\varepsilon, z_{\varepsilon}\right) d x+\left\|z_{\varepsilon}\right\|_{H_{\varepsilon}}^{2} \\
& =\left\|\mathrm{P}_{\varepsilon} Z\right\|_{H_{\varepsilon}}^{2}-2 \int_{\Omega} a\left(\widehat{e}(z)+\partial_{3} z^{1} \otimes_{s} e_{3}\right) \cdot e\left(\varepsilon, z_{\varepsilon}\right) d x+\left\|z_{\varepsilon}\right\|_{H_{\varepsilon}}^{2} \\
& =\left\|\mathrm{P}_{\varepsilon} Z\right\|_{H_{\varepsilon}}^{2}-2\left(\widehat{e}(z)+\partial_{3} z^{1} \otimes_{s} e_{3}, e\left(\varepsilon, z_{\varepsilon}\right)\right)_{L^{2}\left(\Omega ; \mathbb{S}^{3}\right)}+\left\|z_{\varepsilon}\right\|_{H_{\varepsilon}}^{2} \\
& =\left\|\mathrm{P}_{\varepsilon} Z\right\|_{H_{\varepsilon}}^{2}+\left\|e\left(\varepsilon, z_{\varepsilon}\right)-\left(\widehat{e}(z)+\partial_{3} z^{1} \otimes_{s} e_{3}\right)\right\|_{L^{2}\left(\Omega ; \mathbb{S}^{3}\right)}^{2}-\|Z\|_{H}^{2},
\end{aligned}
$$

we have

$$
\left\|\mathrm{P}_{\varepsilon} Z-z_{\varepsilon}\right\|_{H_{\varepsilon}}^{2}-\left(\left\|\mathrm{P}_{\varepsilon} Z\right\|_{H_{\varepsilon}}^{2}-\|Z\|_{H}^{2}\right)=\left\|e\left(\varepsilon, z_{\varepsilon}\right)-\left(\widehat{e}(z)+\partial_{3} z^{1} \otimes_{s} e_{3}\right)\right\|_{L^{2}\left(\Omega ; \mathbb{S}^{3}\right)}^{2},
$$

and the statement follows from condition $\left(T_{2}\right)$.

To prove that $\left(u_{\varepsilon}^{e}\right)$ converges to $U^{e}=\left(u^{e}, u^{e 1}\right)$ in the sense of Trotter, it is enough to show the strong convergence of the scaled strain tensor according to Lemma 1. Like the proof of condition $\left(T_{2}\right)$ of $\mathrm{P}_{\varepsilon}$, we have $e\left(\varepsilon, u_{\varepsilon}^{e}\right) \rightarrow \widehat{e}\left(u^{e}\right)+\partial_{3} u^{e 1} \otimes_{s} e_{3}$ in $L^{2}\left(\Omega ; \mathbb{S}^{3}\right)$. This weak convergence and the fact that $u_{\varepsilon}^{e}, U^{e}$ solve $\left(\mathcal{P}_{\varepsilon}^{e}\right),\left(\mathcal{P}^{e}\right)$ lead to

$$
\begin{aligned}
& \left\|e\left(\varepsilon, u_{\varepsilon}^{e}\right)-\left(\widehat{e}\left(u^{e}\right)+\partial_{3} u^{e 1} \otimes_{s} e_{3}\right)\right\|_{L^{2}\left(\Omega ; \mathbb{S}^{3}\right)}^{2} \\
& \quad=\int_{\Gamma_{\mathrm{N}}} g \cdot u_{\varepsilon}^{e} d \mathcal{H}_{2}-2 \int_{\Omega} a e\left(\varepsilon, u_{\varepsilon}^{e}\right) \cdot\left(\widehat{e}(u)+\partial_{3} u^{e 1} \otimes_{s} e_{3}\right) d x+\int_{\Gamma_{\mathrm{N}}} g \cdot u^{e} d \mathcal{H}_{2} \\
& \quad \rightarrow 2 \int_{\Gamma_{\mathrm{N}}} g \cdot u^{e} d \mathcal{H}_{2}-2 \int_{\Omega} a\left(\widehat{e}(u)+\partial_{3} u^{e 1} \otimes_{s} e_{3}\right) \cdot\left(\widehat{e}(u)+\partial_{3} u^{e 1} \otimes_{s} e_{3}\right) d x=0 .
\end{aligned}
$$


Therefore, $e\left(\varepsilon, u_{\varepsilon}^{e}\right)$ converge strongly to $\widehat{e}\left(u^{e}\right)+\partial_{3} u^{e 1} \otimes_{s} e_{3}$ in $L^{2}\left(\Omega ; \mathbb{S}^{3}\right)$. Note that this convergence is uniform on $[0, T]$.

To prove that $\left(u_{\varepsilon}^{r}\right)$ converges uniformly on $[0, T]$ to $U^{r}=\left(u^{r}, u^{r 1}\right)$ in the sense of Trotter, we have to prove the three conditions of Theorem 1.

Concerning initial data $u_{\varepsilon}^{r 0}$ and $U^{r 0}$, we make an additional assumption on $u_{\varepsilon}^{0}$. That is,

$\left(\mathrm{H}_{3}\right): \quad \exists U^{0}=\left(u^{0}, u^{01}\right)$ in $U^{e}(0)+D(A)$ such that $\left\|\mathrm{P}_{\varepsilon} U^{0}-u_{\varepsilon}^{0}\right\|_{H_{\varepsilon}} \rightarrow 0$.

Hence,

$$
\begin{aligned}
\left\|\mathrm{P}_{\varepsilon} U^{r 0}-u_{\varepsilon}^{r 0}\right\|_{H_{\varepsilon}} & =\left\|\mathrm{P}_{\varepsilon} U^{0}-\mathrm{P}_{\varepsilon} U^{e}(0)-u_{\varepsilon}^{0}+u_{\varepsilon}^{e}(0)\right\|_{H_{\varepsilon}} \\
& \leq\left\|\mathrm{P}_{\varepsilon} U^{0}-u_{\varepsilon}^{0}\right\|_{H_{\varepsilon}}+\left\|\mathrm{P}_{\varepsilon} U^{e}(0)-u_{\varepsilon}^{e}(0)\right\|_{H_{\varepsilon}} .
\end{aligned}
$$

The first term on the right hand side of the inequality goes to zero by the additional assumption on $u_{\varepsilon}^{0}$, while the second term goes to zero, as previously shown.

In regard to the second members $q_{\varepsilon}=\dot{u}_{\varepsilon}^{e}$ and $Q=\dot{U}^{e}$, we again use Lemma 1 by proving $e\left(\varepsilon, \dot{u}_{\varepsilon}^{e}\right) \rightarrow \widehat{e}\left(\dot{u}^{e}\right)+\partial_{3} \dot{u}^{e 1} \otimes_{s} e_{3}$ in $L^{2}\left(\Omega ; \mathbb{S}^{3}\right)$ uniformly on $[0, T]$ because of the smoothness of $g$ with respect to the time $t$.

In the matter of resolvants $\left(I-A_{\varepsilon}\right)^{-1},(I-A)^{-1}$, we have to show that

$$
\forall Y \in X \text {, dense in } H,\left\|\mathrm{P}_{\varepsilon}(I-A)^{-1} Y-\left(I-A_{\varepsilon}\right)^{-1} \mathrm{P}_{\varepsilon} Y\right\|_{H_{\varepsilon}} \rightarrow 0 .
$$

We set $z_{\varepsilon}=\left(I-A_{\varepsilon}\right)^{-1} \mathrm{P}_{\varepsilon} Y$ and $Z=(I-A)^{-1} Y$ that satisfy the problems

$$
\left\{\begin{array}{l}
z_{\varepsilon} \in H_{\varepsilon} \\
\int_{\Omega}(a+b) e\left(\varepsilon, z_{\varepsilon}\right) \cdot e(\varepsilon, v) d x=\int_{\Omega} b e\left(\varepsilon, \mathrm{P}_{\varepsilon} Y\right) \cdot e(\varepsilon, v) d x \quad \forall v \in H_{\varepsilon},
\end{array}\right.
$$

and

$$
\left\{\begin{array}{l}
Z=\left(z, z^{1}\right) \in H ; \\
\int_{\Omega}(a+b)\left(\widehat{e}(z)+\partial_{3} z^{1} \otimes_{s} e_{3}\right) \cdot\left(\widehat{e}(v)+\partial_{3} v^{1} \otimes_{s} e_{3}\right) d x \\
\quad=\int_{\Omega} b\left(\widehat{e}(y)+\partial_{3} y^{1} \otimes_{s} e_{3}\right) \cdot\left(\widehat{e}(v)+\partial_{3} v^{1} \otimes_{s} e_{3}\right) d x \quad \forall V=\left(v, v^{1}\right) \in H .
\end{array}\right.
$$

Now we consider $\left\|\mathrm{P}_{\varepsilon} Z-z_{\varepsilon}\right\|_{H_{\varepsilon}}$. They involve a kind of static problem to which the previous two-fields result on reduction of dimension applies. The proof is then complete and we now have the main theorem.

Theorem 2 Under assumptions $\left(\mathrm{H}_{1}\right)-\left(\mathrm{H}_{3}\right)$, when $\varepsilon$ goes to zero, the sequence of solutions $u_{\varepsilon}$ in $H_{\Gamma_{\mathrm{D}}}^{1}\left(\Omega ; \mathbb{R}^{3}\right)$ to problem $\left(\mathcal{P}_{\varepsilon}\right)$ converges uniformly on $[0, T]$ in the sense of Trotter to the solution $U=\left(u, u^{1}\right)$ in $V_{\mathrm{KL}} \times H^{1}\left(-1,1 ; L^{2}\left(\omega ; \mathbb{R}^{3}\right)\right) / L^{2}\left(\omega ; \mathbb{R}^{3}\right)$ to

$$
(\overline{\mathcal{P}}):\left\{\begin{array}{l}
U=\left(u, u^{1}\right) \in C^{1,1}\left([0, T] ; V_{\mathrm{KL}} \times H^{1}\left(-1,1 ; L^{2}\left(\omega ; \mathbb{R}^{3}\right)\right) / L^{2}\left(\omega ; \mathbb{R}^{3}\right)\right) ; \\
\int_{\Omega}\left[a\left(\widehat{e}(u)+\partial_{3} u^{1} \otimes_{s} e_{3}\right)+b\left(\widehat{e}(\dot{u})+\partial_{3} \dot{u}^{1} \otimes_{s} e_{3}\right)\right] \cdot\left(\widehat{e}(v)+\partial_{3} v^{1} \otimes_{s} e_{3}\right) d x \\
\quad=\int_{\Omega} f \cdot v d x+\int_{\Gamma_{\mathrm{N}}} g \cdot v d \mathcal{H}_{2} \\
\forall V=\left(v, v^{1}\right) \in V_{\mathrm{KL}} \times H^{1}\left(-1,1 ; L^{2}\left(\omega ; \mathbb{R}^{3}\right)\right) / L^{2}\left(\omega ; \mathbb{R}^{3}\right) .
\end{array}\right.
$$


Hence $U$ solves a problem of visco-elasticity with short memory of Kelvin-Voigt type, but involving a couple $\left(u, u^{1}\right)$ of state variables. Clearly the field $u^{1}$ can be eliminated as in [1] so that $u$ does solve a problem of visco-elasticity with long (but fading) memory.

\section{Acknowledgements}

The first author was supported by DPST scholarship (Development and Promotion of Science and Technology Talents Project) for doing this research.

\section{Funding}

The first author was supported by DPST scholarship (Development and Promotion of Science and Technology Talents Project) for doing this research.

\section{Competing interests}

The authors declare that they have no competing interests.

\section{Authors' contributions}

$\mathrm{CL}$ conceived of the research work and verified the result. SO and YT proved the main result, interpreted the result and wrote a paper. All authors read and approved the final manuscript.

\section{Authors' information}

Yotsawat Terapabkajornded, email: ongyosawat@gmail.com; Somsak Orankitjaroen, email: somsak.ora@mahidol.ac.th; Christian Licht, email: christian.licht@umontpellier.fr.

\section{Author details}

${ }^{1}$ Department of Mathematics, Faculty of Science, Mahidol University, Bangkok, Thailand. ${ }^{2}$ LMGC-UMR 5508, Université de Montpellier-CC048, Montpellier, France. ${ }^{3}$ Centre of Excellence in Mathematics, CHE, Bangkok, Thailand.

\section{Publisher's Note}

Springer Nature remains neutral with regard to jurisdictional claims in published maps and institutional affiliations.

Received: 29 January 2019 Accepted: 14 April 2019 Published online: 14 May 2019

\section{References}

1. Licht, C.: Thin linearly visco-elastic Kelvin-Voigt plates. C. R., Méc. 341, 697-700 (2013)

2. Licht, C., Weller, T.: Approximation of semi-groups in the sense of Trotter and asymptotic mathematical modeling in physics of continuous media. Discrete Contin. Dyn. Syst., Ser. S 12, 1709-1741 (2019)

3. Ciarlet, P.G.: Mathematical elasticity, vol. II. In: Lions, J.L., Papanicolaou, G., Fujita, H., Keller, H.B. (eds.) Study in Mathematics and Its Applications, vol. 27, pp. 14-80. North-Holland, Amsterdam (1997)

\section{Submit your manuscript to a SpringerOpen ${ }^{\circ}$ journal and benefit from:}

- Convenient online submission

- Rigorous peer review

- Open access: articles freely available online

- High visibility within the field

- Retaining the copyright to your article 